\title{
Analysis of sports dance major affect for development influence
}

\author{
Shi Rui Shao ${ }^{1, a^{*}}$ and Wen Lan Lian ${ }^{1}$ \\ ${ }^{1}$ Faculty of Sports Science, Ningbo University, Ningbo China \\ ahaoqinb@aliyun.com
}

Keywords: Sports dance; Influence factors; Principal component analysis; Linear combination; Weight

\begin{abstract}
Nowadays material civilization and spiritual civilization of human beings have been rapidly developing; sports dance has become a sports event that is favored by people. Due to China covers a vast geographic area and has a large population, sports dance development suffers many factors influence as economic level is limited, people acceptance levels are not enough and so on.When research on sports dance development relative problems, excessive such factors will lead to inconvenience in researching. The paper takes sports dance development as research objects, takes teachers, referees, athletes' three types of population questionnaire survey results as evidence, targeted at economic level, competition system, scientific research level and other thirteen influence factors to make principal component analysis. Analysis result indicates that two main components can replace the thirteen influence factors. The two principal components are three influence factors' linear combination, the differences between the two is that every factor weight is different.
\end{abstract}

\section{Introduction}

In recent years, sports dance has been developed in lots of universities, and become one of well-received sports events among numerous university students. To this day, sports dance development is still not balanced. The problem leads to people to think about sports dance development constraint factors.

Competition organizations were disordered, management was poor, and these factors serious restricted Hebei province sports dance development [1-3]. In 2004, Zheng Chuan-Feng and others in the article "Research on sports dance development and countermeasures in universities", they made comprehensive analysis of present sports dance development status in universities. Result showed, each university sports dance development levels were different, students' interests and positivity in sports dance event were higher, but overall they presented as backward of theoretical knowledge and scientific researches [4-7]. With respect to this, authors provided countermeasures. In order to let sports dance to be able to better develop, author pointed out, it should propel to sports dance optional course and club-oriented [8-10]. In 2012, Bi Fei in the article "University sports dance event education research", applied teaching experiment method and others multiple research methods, analyzed universities sports dance teaching transforming towards sports dance education issues, result showed that sports dance education functions were not only letting students to master sports dance basic motions, but also can let students' attainments and quality to be improved [10-12]. Sports dance event teaching conformed to the trends of times that were worth promoting to each university. In 2003, Zhao Li in the article "Chinese sports dance organization status and counter measure research", applied multiple research methods, researched on sports dance development influence factors, research result showed Chinese sports dance lacked of self-textbook system, scientific research levels were lower, referees grade evaluation system was not normalized, referees education degrees were generally lower [13]. This paper takes Chinese university sports dance development status as research object, analyzes teachers, referees and athletes each kind of situations, and further gets conclusion.

\section{Methods}

The model researches on sports dance development influence factors, takes each factor teachers occupied percentage, referee occupied percentage and athlete occupied percentage as evidence, 
and reduces multiple influence factors into fewer influence factors so as to easy for the kind of problems' late researching. Table 1 data is from 'Chinese sports dance development status investigation and countermeasure research'.

Table 1 Original data table

\begin{tabular}{cccccccc}
\hline Influence factor & Teacher & Percentage\% & Referee & Percentage $\%$ & Athlete & Percentage $\%$ & Rank \\
\hline Economic level constraints & 132 & 80 & 22 & 43 & 630 & 61 & 8 \\
Competition and referee system & 140 & 84 & 37 & 73 & 932 & 90 & 3 \\
Teachers level & 154 & 93 & 51 & 100 & 1000 & 97 & 1 \\
Funding issue & 93 & 56 & 43 & 84 & 403 & 39 & 11 \\
Related to non-Olympic Games events & 103 & 62 & 41 & 80 & 726 & 70 & 4 \\
Fewer international exchange & 149 & 90 & 47 & 92 & 955 & 92 & 2 \\
Public concept & 57 & 34 & 30 & 59 & 745 & 72 & 6 \\
Scientific research level & 73 & 44 & 25 & 49 & 352 & 34 & 12 \\
Sports level & 62 & 37 & 32 & 63 & 601 & 58 & 9 \\
Mass media influence & 88 & 53 & 30 & 59 & 722 & 70 & 5 \\
Disordered organizational management & 77 & 46 & 24 & 47 & 561 & 54 & 10 \\
Field facilities & 96 & 58 & 17 & 33 & 677 & 65 & 7 \\
Others & 26 & 16 & 6 & 12 & 135 & 13 & 13 \\
\hline
\end{tabular}

\section{Results}

By such rotating, every factor's significance is relative clear. From the table, it can see that two main factors are extracted. From Fig. 1, it can more intuitive indicate.

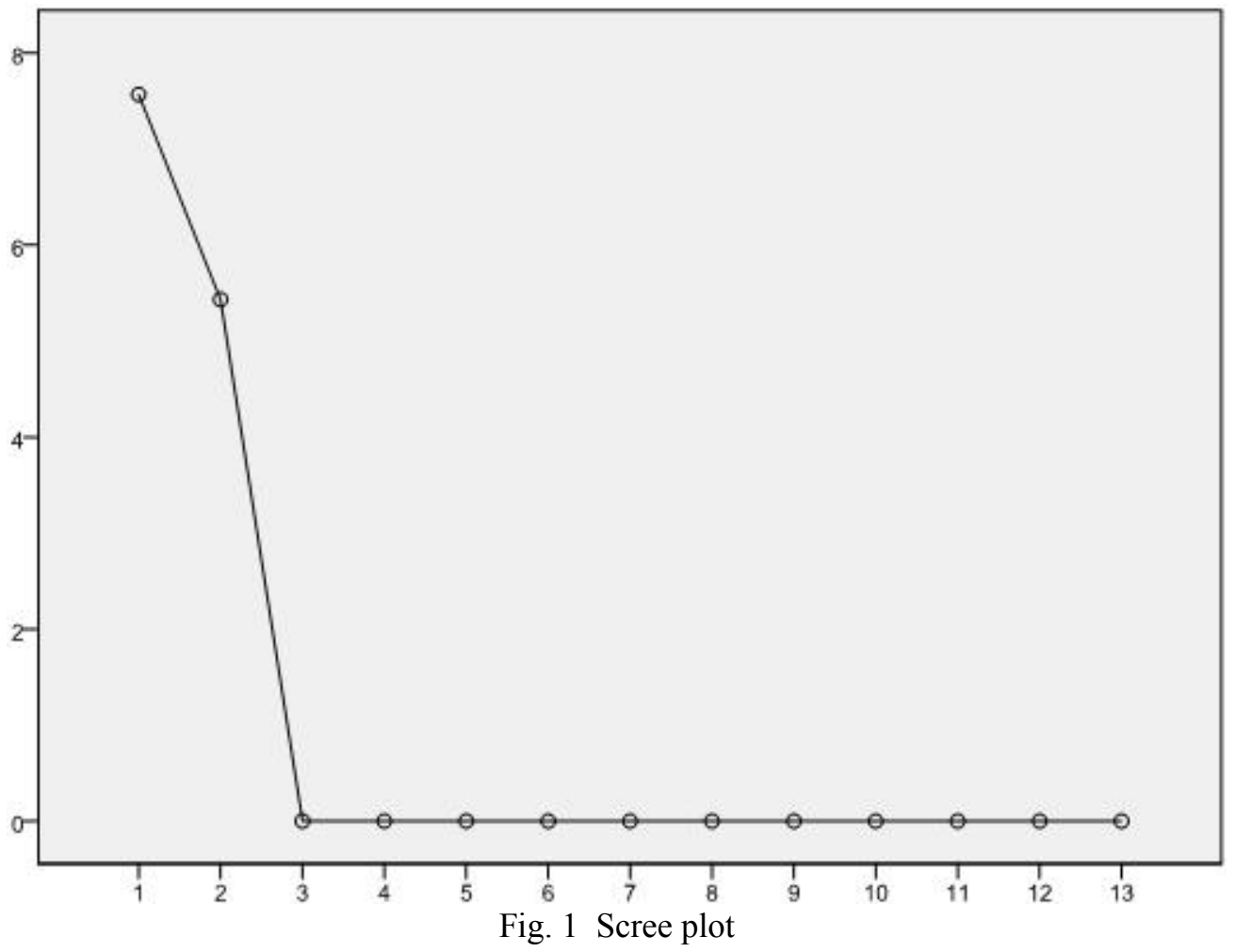

Figure 1 is feature values' scree plot. In general, the figure shows big factor steep slope and surplus factor gentle tail has obvious interruption. Generally selected main factors are in the very steeply slope, and factors lie in gentle slope have insignificant effects on total. From Figure 1, it is clear that the former two factors are in the relative steeply slope, and starts from the third factor, the slope turns to be gentle, while starts from the third factor, the slope is nearly zero, therefore select two factors as comprehensive factors. 


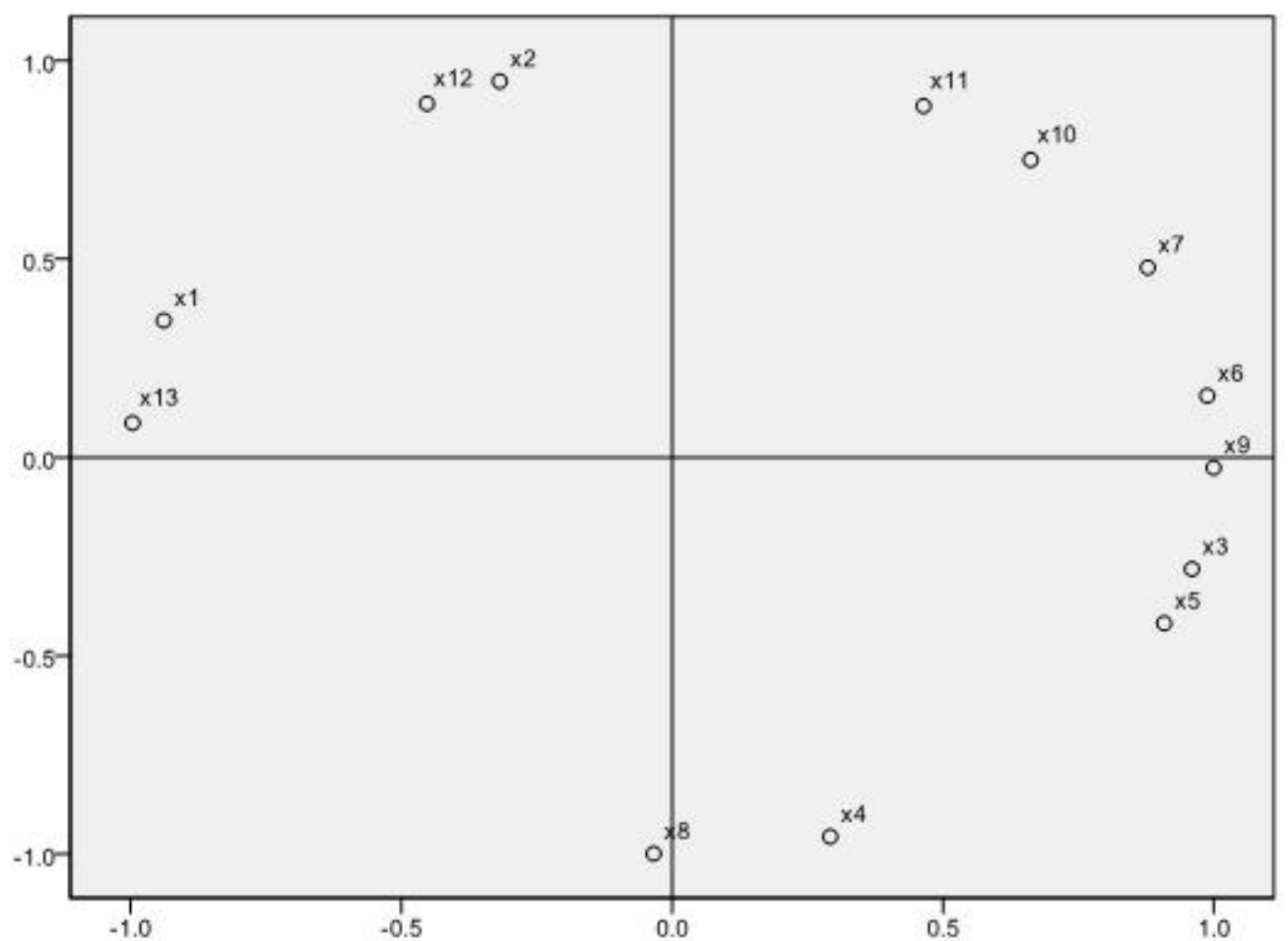

Fig.2 The view of the rotating components of the space

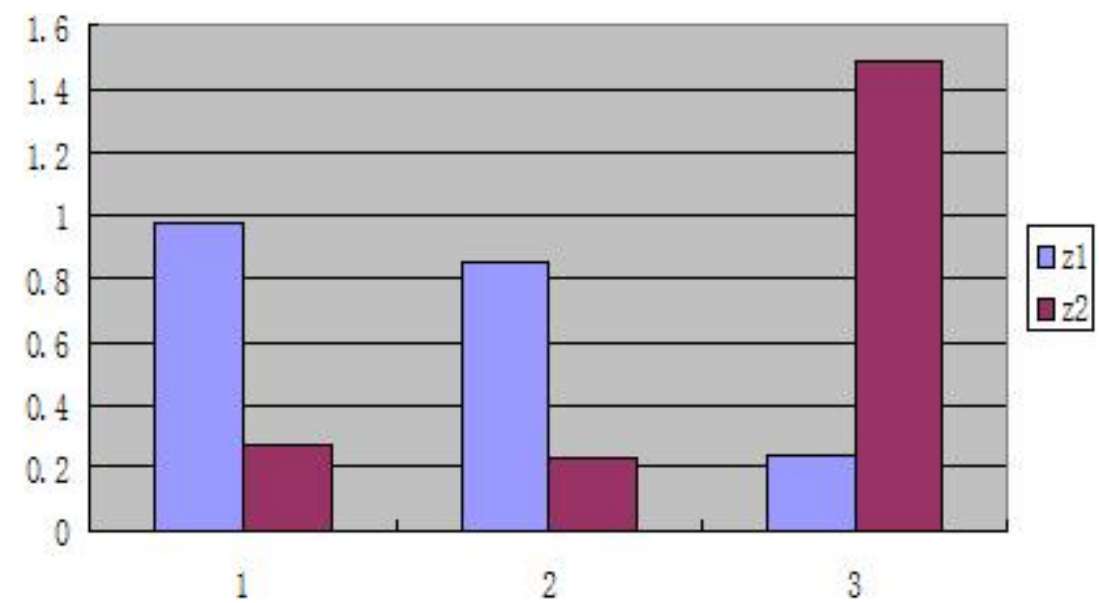

Fig. 3 Principal component comparison chart

Figure 4 is principal component comparison chart. From Figure 4, it is clear that to teachers and referees, principal component 1 occupied weight is bigger than that of principal component 2 , to athletes; principal component 1 occupied weight is smaller than that of principal component 2 . From Fig. 2, it is clear that principal component analysis totally extracts two main factors this time, that get closer to coordinate axis have big factor loading and explanatory ability is relative strong. After defining main factors amount, it should calculate feature vectors, feature vectors amount is the same as main factors amount. Feature vector matrix is as Table 2 show.

Table 2 Feature vector matrix

\begin{tabular}{cc}
\hline F1 & F2 \\
\hline-1.58831 & -0.42853 \\
-1.01902 & 0.98213 \\
1.03123 & -0.32397 \\
0.42783 & -1.62071 \\
1.01548 & -0.5518 \\
0.91186 & 0.3439 \\
0.65869 & 0.78239 \\
0.03037 & -1.82201 \\
0.99056 & 0.0755 \\
0.28878 & 1.09916 \\
-0.00898 & 1.21932 \\
-1.16855 & 0.84169 \\
-1.56994 & -0.59705 \\
\hline
\end{tabular}




\section{Conclusion}

Utilize principal component analysis thought to use fewer variables to replace original multiple variables, these fewer variables can reflect original data most information. In addition, the model more focuses on information comprehensive evaluation. The method also has certain drawbacks, such as, when principal component factor loading positive and negative symbols are simultaneously existing, evaluation function significances will not be clear, naming clarity will be low, only involve a group of variables' correlations. The model's princi pal component is composed of original factors linear combinations, so principal components actual significances are hard to define, just functions as dimension reduction. Principal component analysis application field is very widely, such as "regional water resources carrying capacity problem ", "Town land evaluation problem", "3G network comprehensive performance evaluation problem" and o ther aspects' analysis problems. The paper applies principal component analysis into sports dance development restriction factors, succeeds in reducing three influence factors into two principal components, and is convenient for later such kinds of problems researching.

\section{References}

[1] Wang Xiaoguang. Research On Development, 2007, (5), 57-60.

[2] CHEN Nan - yue. Journal of Yunnan Finance and Trade Institute, 2005, 21(6), 81-85.

[3] LIU Chang-ming, GUAN Bin. Journal of Tianjin University(Social Sciences), 2009, 11(5), 453-457.

[4] Fan Liwei, Liang Jiyao. Value Engineering, 2006, 25(5), 29-31.

[5] SUN Yi-kai. Journal of Anhui University(Philosophy \& Social Sciences), 2006, 30(6).

[6] LU Jian-hua. Journal of Anhui University(Philosophy \& Social Sciences), 2006, 30(6), 5-6.

[7] XU Xiao-yue. Journal of Anhui University(Philosophy \& Social Sciences), 2006, 30(6), 6-8.

[8]SU Bao-mei, LIU Zong-xian, Liu Chang-ming. Journal of Jinan University, 2002, 12(5), 1-10.

[9] Zhang B.; Zhang S.; Lu G.. Journal of Chemical and Pharmaceutical Research, 2013, 5(9), 256-262.

[10] Zhang B.; International Journal of Applied Mathematics and Statistics, 2013, 44(14), 422-430.

[11] Zhang B.; Yue H.. International Journal of Applied Mathematics and Statistics, 2013, 40(10), 469-476.

[12] Zhang B.; Feng Y.. International Journal of Applied Mathematics and Statistics, 2013, 40(10), 136-143.

[13] Bing Zhang. Journal of Chemical and Pharmaceutical Research, 2014, 5(2), 649-659. 